\title{
In-Situ Monitoring of Photoresist Thickness Contour
}

\author{
Weng Khuen Ho, Xiaodong Wu and Arthur Tay \\ Department of Electrical and Computer Engineering \\ National University of Singapore,119260 Singapore \\ elehowk@nus.edu.sg
}

\author{
Xiaoqi Chen \\ Singapore Institute of Manufacturing Technology \\ 71 Nanyang Drive Singapore 638075 \\ xqchen@SIMTech.a-star.edu.sg
}

\begin{abstract}
In microelectronics processing, coating of photoresist is a common process. It is important to ensure the uniformity of the photoresist thickness across the wafer. In this paper, we propose an in-situ monitoring system. In the setup, a spectrometer is used to measure the photoresist thickness contour on the wafer after the spin-coat step or edge-bead removal step. The experimental results are compared with off-line ellipsometer measurements. The worst-case error is experimentally found to be less than $2 \%$.
\end{abstract}

\section{INTRODUCTION}

As the feature size of semiconductor devices decrease dramatically, the challenge to accomplish efficient monitoring of process status in the semiconductor manufacturing process becomes more difficult [1]. One of the key parameters is the resist thickness contour on the wafer because of its impact on the critical dimension (CD) uniformity across wafer [2]. Currently, the industry is also moving towards the larger 300 $\mathrm{mm}$ wafers for economic reasons and uniformity tends to deteriorate for a larger surface area.

In a streamlined process flow of microelectronics production, there is tremendous benefit to perform in-situ monitoring so that defective wafer can be detected early. Rectifications can be made before the defective wafer proceeds to downstream processes incurring unnecessary costs. Our objective is to develop an in-situ contour monitoring system such that a wafer once coated with photoresist can immediately be inspected to determine its suitability for subsequent processes.

There have been some research on in-situ monitoring of the resist thickness and properties. To study the bake mechanism, Paniez et al [3] used in-situ ellipsomtery while Fadda et al [4] used contact angle measurements to monitor the resist thickness. Morton et al [5] used in-situ ultrasonic sensors to monitor the change in resist properties to determine whether the resist has been sufficiently cured, thereby determining the endpoint of the softbake process. In related work, Lee et al [7] used in-situ multiwavelength reflection spectrometer to measure the resist thickness versus bake time and then real time feedback control applied to improve thickness uniformity. In [6], a plastic-fiber-bundle probe array was applied to monitor the photoresist thickness change at multiple sites for developing rates and develop endpoint.

In contrast with the multiple-probe instrument [6], we propose a new instrumentation that uses only one probe attached to a linear slider to monitor resist contour on the wafer.

Typically the coating process involves the spraying of photoresist on a spinning wafer before transfering to another station to remove the edge beads that have built up during the spin coat process. Edge bead removal is performed immediately after spin coat by directing a stream of remover near the edge of the wafer while it is spinning. In both processes a spectrometer attached to a linear slider mounted above the spinning wafer can be used to take measurements to give the contour of the photoresist thickness. For convenience, readings are taken at low speed. Specifically, measurement can be taken at the end of the spin-coat process when the spinning slows down to stop. Alternatively, measurements can be taken at low spinning speed before and/or after the dispensing of the edge bead removal when spinning begins and ends.

The paper is organized as follows. The setup and theorectical analysis are described in Sections II and III respectively. The algorithm is given in Section IV and experimental results in Section V. Finally, conclusions are given in Section VI.

\section{EXPERIMENTAL SETUP}

The experimental setup used to demonstrate the concept consists of three main parts as shown in Figure 1: a motor to spin the wafer, a spectrometer attached to a linear slider and a computing unit. The photo is shown in Figure 2. In all experiments, commercial DUV resist Shipley SL4000 was spin-coated on an 8-inch wafer. The thickness profile across the wafer was monitored.

The spectrometer probe was attached to a linear slider mounted above the spinning wafer and moved back and forth continuously between the center and edge of the wafer. The sliding speed can be adjusted to monitor different points on the wafer.

The spectrometer had a setup similar to the multiwavelength DRM [11]. It comprised a broadband light source (LS-1), a spectrometer with the capability of monitoring the reflected light intensity(S2000), and a bifurcated fiber optics reflection probe consisting of a bundle of 7 optical fibers (6 illumination fibers and around 1 read fiber) positioned above the wafer to monitor the resist thickness in real time. During the monitoring process, light from the broadband light source was focused on the resist through one end of the probe and the reflected light was guided back to the spectrometer through the other end. 


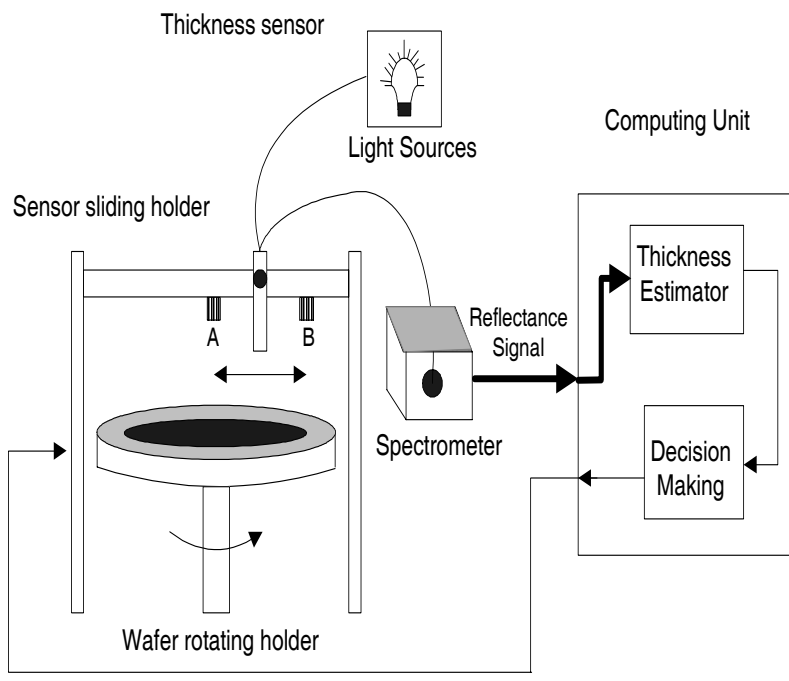

Fig. 1. Experimental setup

The reflectance signals were acquired through the A/D converter and the computing unit converted them to thickness measurements using a thickness estimation algorithm in a LabView environment. The thickness estimation algorithm is discussed in Section IV. With the availability of the entire thickness profile across the wafer, proper assessment and rectification actions can be taken.

\section{Reflection of Light b y Moving Medium}

Reflection of electromagnetic wave by a moving medium has been investigated by numerous researchers. For a detailed review readers can refer to [13]. Two factors affects a moving medium reflectivity measurement: reflection angle and reflection coefficient. For reflection angle, the ordinary law of reflection may not be valid when the plane is moving. Consider the construction of Figure 3 and the equation below [14] which gives the relationship between the incident angle $\alpha$ and reflected angle $\beta$ as a function of the relative moving velocity $v$

$$
\frac{\sin \alpha-\sin \beta}{\sin (\alpha+\beta)}=\frac{v}{c} \sin \varphi
$$

where $c$ is the speed of light in air. In the experimental setup, $\varphi=0$ for normal incidence of light. Hence the ordinary law of reflection $\alpha=\beta$ holds, which means that the reflected light of normal incident light is still normal.

Next, we consider the reflection coefficient. If the incident light is not normal to the moving plate then the reflection coefficient expression is complex. But for the experimental setup, the incident light is normal to the moving wafer. The reflection coefficient is given as [15]

$$
r=-\frac{n^{2}-\sqrt{\frac{n^{2}-\gamma^{2}}{1-\gamma^{2}}}}{n^{2}+\sqrt{\frac{n^{2}-\gamma^{2}}{1-\gamma^{2}}}}
$$

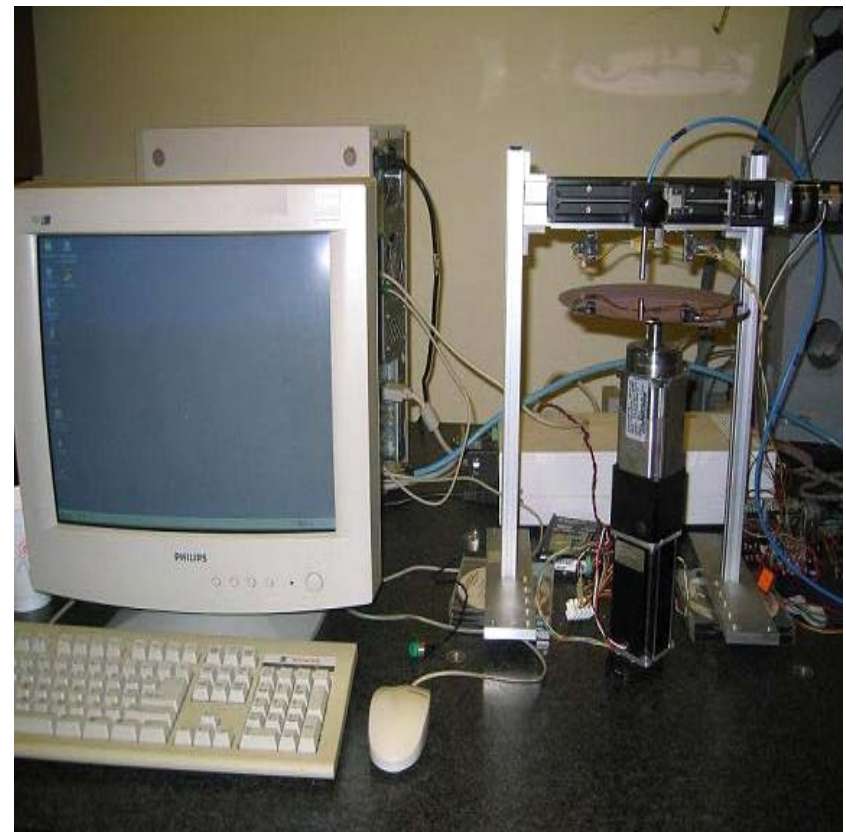

Fig. 2. Photograph of experimental setup

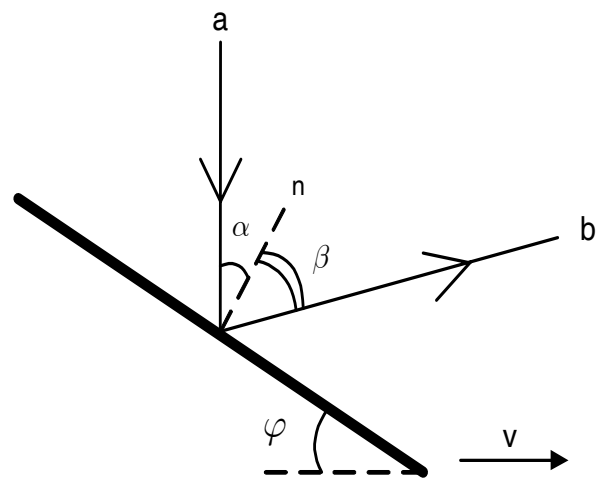

Fig. 3. The plate is moving at a constant speed of $v$. The incident light, reflected light, angle of incidence and angle of reflection are given as $a, b$, $\alpha$ and $\beta$. respectively

where $n$ is the refractive index of the moving medium, and $\gamma=\frac{v}{c}$. For a stationary medium or slow moving medium $(v \approx 0)$, Equation $(2)$ can be simplified to the familiar equation

$$
r=\frac{1-n}{1+n}
$$

Combined with the thin film reflectivity given in Equation (5), the influence of speed on the spectral reflectivity curve can be computed as shown in Figure 4. From this figure, we can see that at low speed $(\gamma<0.1)$ the spectral reflectivity is almost the same as the stationary case. Only at high speed, the spectral reflectivity curve deviates noticeably from its stationary counterpart.

\section{THICKNESS ESTIMATION}

The spectrometer is positioned above the wafer coated with a layer of photoresist to measure the resist thickness 


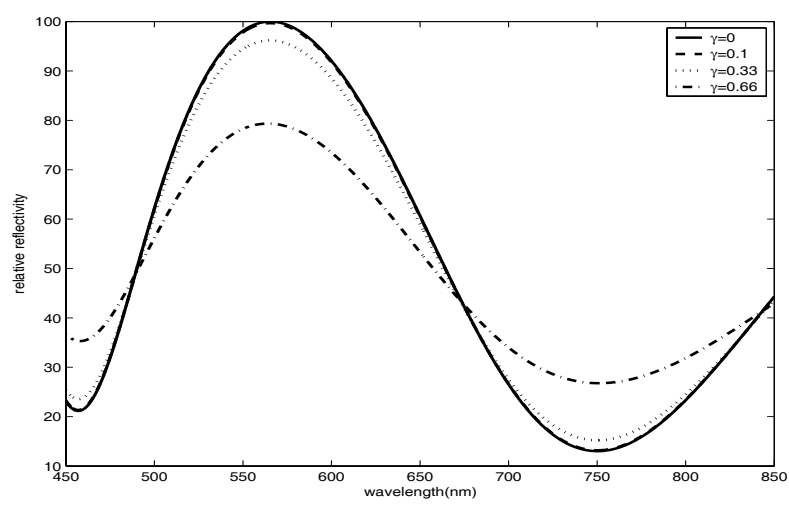

Fig. 4. The effect of speed on the spectral reflectivity curve where $\gamma=\frac{v}{c}$ and $v$ is the speed of the moving plate and $c$ the speed of light.

[10]. We can measure the reflectance signals in the wavelength range from $450 \mathrm{~nm}$ to $850 \mathrm{~nm}$. Photons at low wavelength will incur unwanted photoresist exposure, so proper optical filter may have to be attached to eliminate low wavelength range.

The resist thickness $y$ can be derived from a thin-film optical model. Based on discussion in Section III, at low speed the spectral reflectivity curve can be approximated by the stationary curve. Consider a photoresist film with a refractive index of $n_{r}$. Its relation with the reflectance intensity is given in [12] as

$$
h(\lambda, y)=\frac{r_{12}^{2}+r_{23}^{2}+2 r_{12} r_{23} \cos (2 \beta)}{1+r_{12}^{2} r_{23}^{2}+2 r_{12} r_{23} \cos (2 \beta)}
$$

where

$$
r_{12}=\frac{n_{a}-n_{r}}{n_{a}+n_{r}} \quad r_{23}=\frac{n_{s}-n_{r}}{n_{s}+n_{r}} \quad \beta=\frac{2 \pi n_{r} y}{\lambda}
$$

and $n_{a}, n_{r}$ and $n_{s}$ are the refractive index of air, resist, and silicon substrate respectively. Here the equation $r_{12}=\frac{n_{a}-n_{r}}{n_{a}+n_{r}}$ reduced to Equation (3) since the refractive index of air is 1. $y$ is the resist thickness. The variation of the resist refractive index with wavelength $\lambda$ is given by the Cauthy equation [12]

$$
n_{r}(\lambda)=A+\frac{B}{\lambda^{2}}+\frac{C}{\lambda^{4}}
$$

where $A, B, C$ are the Cauchy parameters of the resist. The $A, B, C$ parameters for resist are obtained from resist vendor. For the case of Shipley SL4000 the parameters are $A=1.546 \mathrm{~nm}, B=5.9 \times 10^{3} \mathrm{~nm}$, and $C=6 \times 10^{8} \mathrm{~nm}$. The spectral refractive index of silicon can also be obtained from a spectral model at room temperature [8].

Given the reflectance measurements, the resist thickness can be estimated using Equation (4). For a fairly repeatable process, we can give a good initial estimate of the thickness. To meet the demand of real-time monitoring, we use the least square estimation method to estimate the resist thickness[7]. To do so, Equation (4) is approximated by taking Taylor series expansion to give

$$
h(\lambda, y)=h\left(\lambda, y_{0}\right)+\left.\frac{\partial h}{\partial y}\right|_{\lambda, y_{0}} \delta y
$$

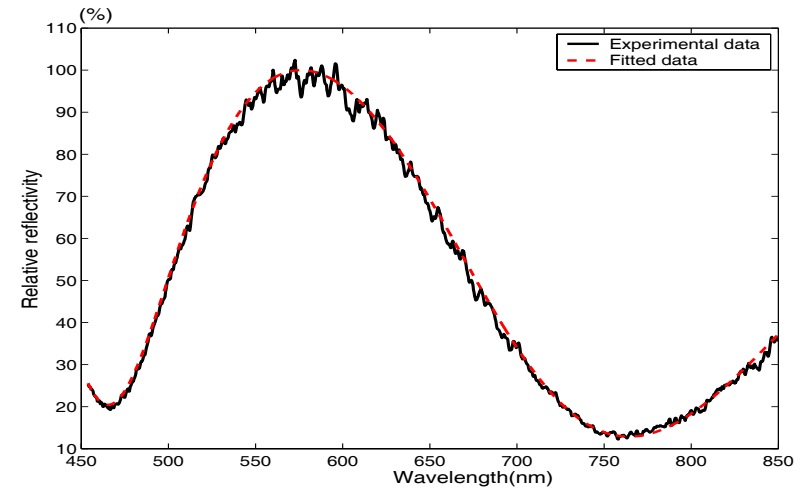

Fig. 5. Fitting of optical model with experimental data. Experimental data: solid-line; model: dashed-line.

where $y_{0}$ is the initial coefficient estimate and $\partial h / \partial y$ the derivative. The estimated thickness is then given as

$$
\hat{y}=y_{0}+\delta y
$$

and the change in resist thickness $\delta y$ is estimated using the least square method given by

$$
\delta y=\left(\frac{\partial h}{\partial y}^{T} \frac{\partial h}{\partial y}\right)^{-1} \frac{\partial h}{\partial y}^{T}\left(y-y_{0}\right)
$$

where

$$
\begin{gathered}
\frac{\partial h}{\partial y}=\left[\begin{array}{c}
\left.\frac{\partial h}{\partial y}\right|_{\lambda_{1}, y_{0}} \\
\left.\frac{\partial h}{\partial y}\right|_{\lambda_{2}, y_{0}} \\
\ldots \\
\left.\frac{\partial h}{\partial y}\right|_{\lambda_{M}, y_{0}}
\end{array}\right] \quad h=\left[\begin{array}{c}
h\left(\lambda_{1}, y\right) \\
h\left(\lambda_{2}, y\right) \\
\ldots \\
h\left(\lambda_{M}, y\right)
\end{array}\right] \\
h_{0}=\left[\begin{array}{c}
h\left(\lambda_{1}, y_{0}\right) \\
h\left(\lambda_{2}, y_{0}\right) \\
\ldots \\
h\left(\lambda_{M}, y_{0}\right)
\end{array}\right]
\end{gathered}
$$

To estimate the resist thickness, reflectance measurements were obtained at wavelength between $450 \mathrm{~nm}$ and $850 \mathrm{~nm}$, about $0.35 \mathrm{~nm}$ apart. For setup a sample rate of $0.16 \mathrm{~s}$ was used. Results from the optical model is compared with experimental data in Figure 5.

\section{IN-SITU MONITORING}

\section{A. Trajectory}

The trajectory to monitor is decided by the combination of wafer spinning speed and spectrometer sliding speed. Denote $T_{R}$ as the time for one revolution of the wafer and $T_{C E}$ the time for the sensor probe to move from centre to edge of the wafer or vice versa. Define the ratio of period as

$$
\eta=\frac{T_{R}}{T_{C E}}
$$

The ratio, $\eta$ decides the trajectory monitored on the wafer surface. In the experiments we set $T_{R}=6 \mathrm{~s}, T_{C E}=1.3 \mathrm{~s}$ and sampling time of 0.16 s giving $\eta=4.5$. The trajectory is shown in Figure 6 and the points on the wafer with readings taken are marked with asterisks. 


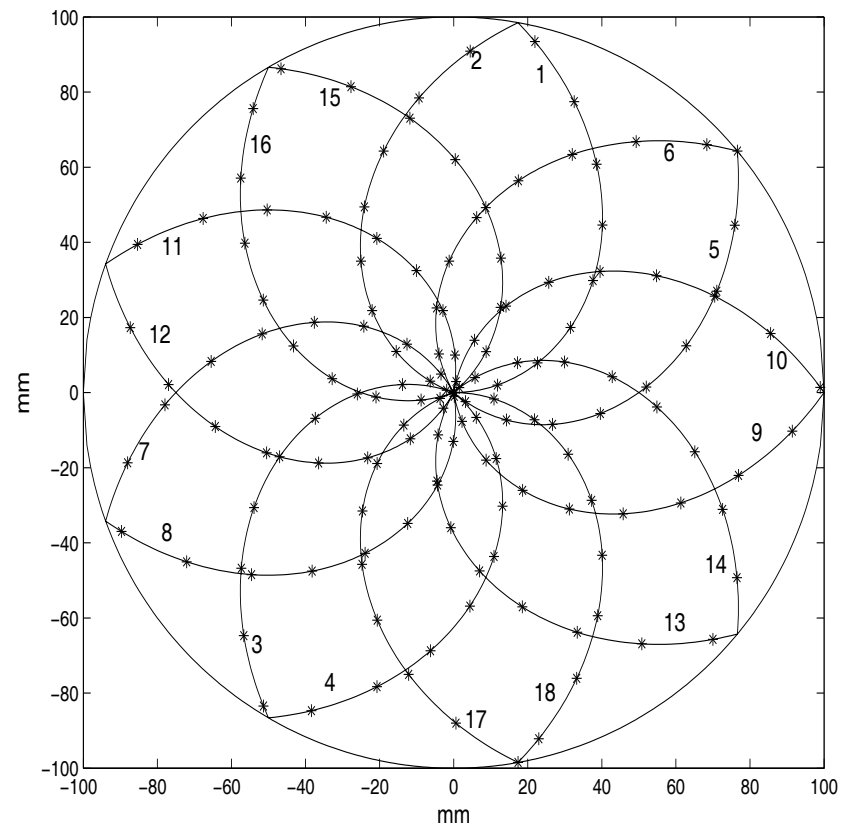

Fig. 6. The points on the wafer with measurements taken are marked with asterisks.

\section{B. Experimental Result}

An 8-inch wafer was coated with a layer of Shipley SL4000 photoresist. The contour and the 3-D profile are shown in Figures 7 and 8. From the thickness profile, we would be able to evaluate the coating quality across the whole wafer. Statistical quality such as thickness mean and standard deviation can be calculated as well. For this test wafer, photoresist at the centre was thicker than the edge. The statistical mean $\bar{y}=371.45 \mathrm{~nm}$ and standard deviation $\sigma=9.25 \mathrm{~nm}$. Based on these information, simple accept/reject action or advanced run-to-run control scheme can then be applied and in turn possibly lead to cost saving and manufacturing performance improvement.

\section{Comparison with off-line ellipsometer}

The thickness measurements obtained from the experiments were compared with those from an off-line spectroscopic ellipsometer measurement. Ellipsometry is an optical technique devoted to the analysis of surfaces based on the measurement of the variation of the polarization state of the light after reflection on a plane surface. The thickness measurements from the ellipsometer are compared with those from the experiments. Both single point measurement and statistical results are compared with good agreement. The comparison results are shown in Table I. The positioning of P1, P2, P3, P4, P5 are shown in Figure 9. The worst-case percentage error is less than $1 \%$. Further experiments on two more wafers gave a worst-case percentage error of less than $2 \%$.

\section{Conclusion}

In microelectronics processing, coating of photoresist is a common process. It is important to ensure the uniformity of

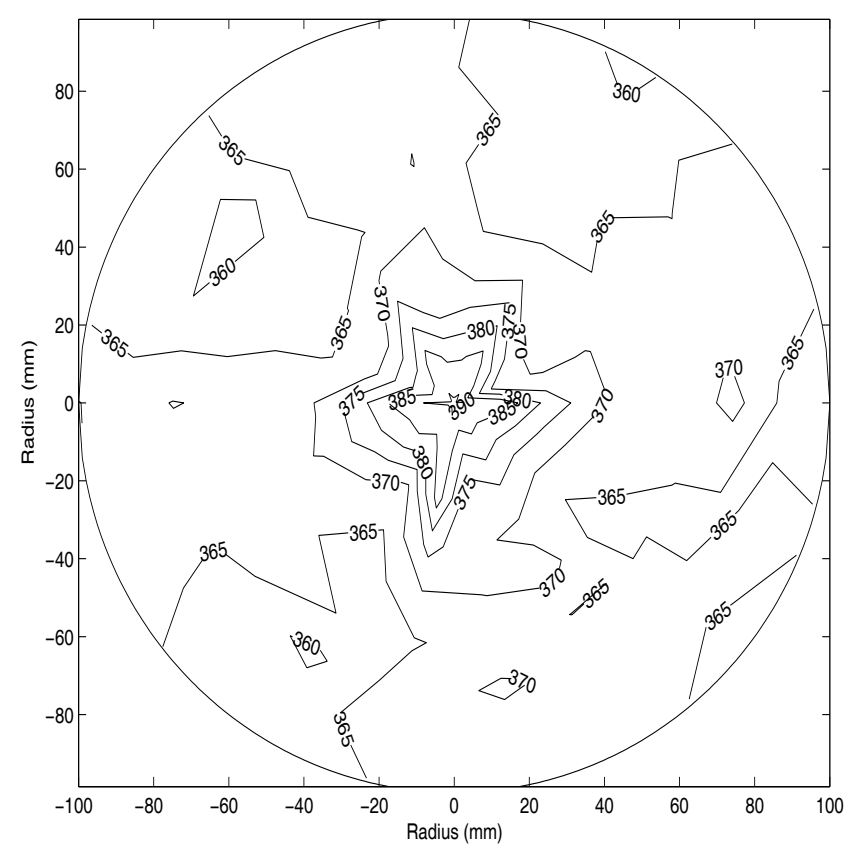

Fig. 7. Resist thickness contour

TABLE I

COMPARISON OF IN-SITU MEASUREMENTS WITH OFF-LINE ELLIPSOMETER MEASUREMENTS

\begin{tabular}{|c||c|c|c|c|c||}
\hline & P1 & P2 & P3 & P4 & P5 \\
\hline $\begin{array}{c}\text { Off-line } \\
\text { Ellipsometer } \\
(\mathrm{nm})\end{array}$ & 390.29 & 363.51 & 366.74 & 364.12 & 366.48 \\
\hline $\begin{array}{c}\text { In-situ } \\
\text { measurement } \\
(\mathrm{nm})\end{array}$ & 389.12 & 365.07 & 365.38 & 364.95 & 363.53 \\
\hline $\begin{array}{c}\text { Percentage } \\
\text { error } \\
(\%)\end{array}$ & 0.3 & -0.4 & 0.4 & -0.2 & 0.8 \\
\hline
\end{tabular}

the photoresist across the wafer. In this paper, we proposed an in-situ monitoring system. In the setup, a spectrometer was used to measure the photoresist thickness contour on the wafer after the spin-coat step or edge-bead removal step. The experimental results were compared with offline ellipsometer measurements. The worst-case error was experimentally found to be less than $2 \%$.

\section{REFERENCES}

[1] International Technology Roadmap for Semiconductor 2004 Update: Lithography, Semiconductor Industry Assn., 2004, p. 6.

[2] J. R. Sheats and B. W. Smith, Microlithography: Science and Technology. New York: Marcel Dekker, 1998, pp. 31-33.

[3] P. J. Paniez, A. Vareille, P. Ballet, and B. Mortini, "Study of bake mechanisms by real-time in-situ ellipsometry," Proc. SPIE, vol. 3333, 1998, pp. 289-300.

[4] E. Fadda, C. Clarisse, and P. J. Paniez, "Study of bake mechanisms in novolak based photoresist films: Invetigation by contact angle measurements," Proc. SPIE, vol. 2714, 1996, pp. 460-468.

[5] S. L. Morton, F. L. Degertekin, and B. T. Khuri-Yakub, "Ultrasonic monitoring of photoresist processing," Proc. SPIE, vol. 3677, 1999, pp. 340-347.

[6] G.R. Lin, "Dynamical mapping and end-point detection of photoresist development by using plastic-fiber bundle probe arry", IEEE Trans. instrum. meas., vol. 48, no. 6, 1999, pp. 1319-1323. 


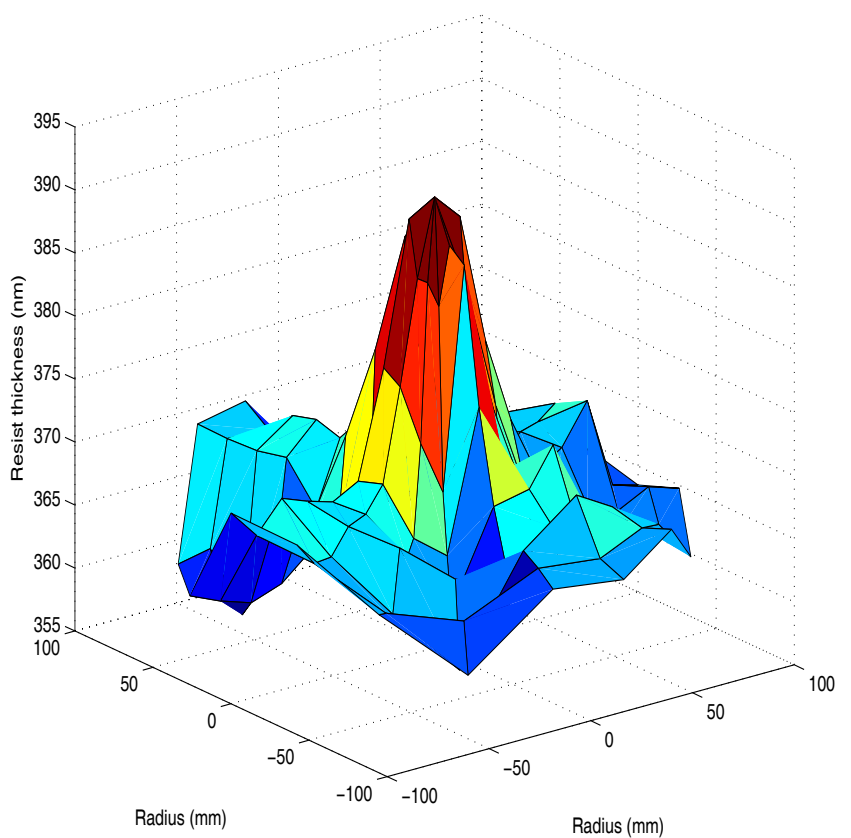

Fig. 8. Resist thickness: 3-D representation.

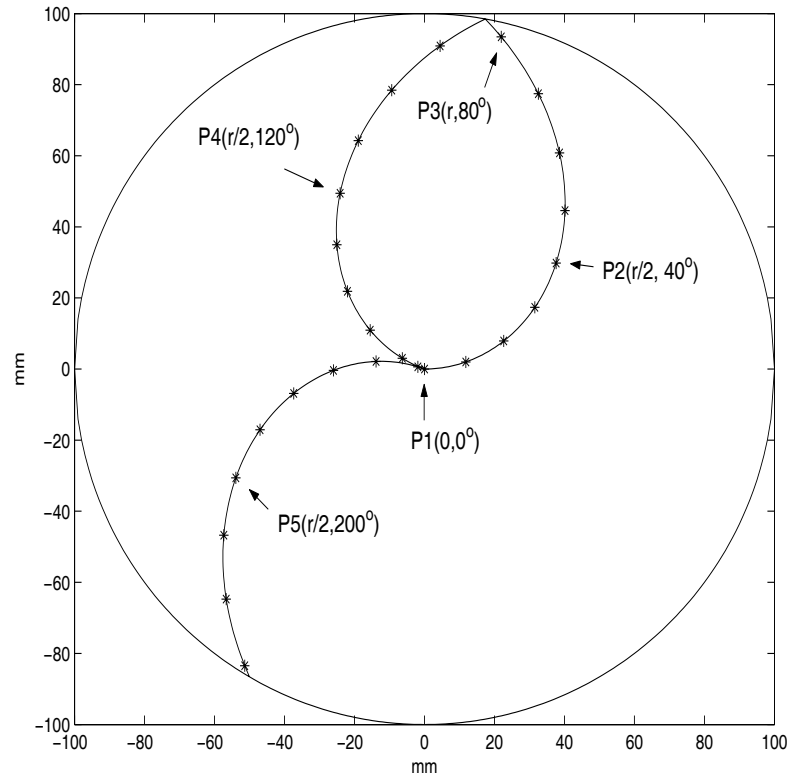

Fig. 9. Five points in Figure 6 were compared with off-line ellipsometer measurements.

[7] L. L. Lee, C. D. Schaper, and W. K. Ho, "Real-time predictive control of photoresist Film thickness uniformity," IEEE Trans. Semiconduct. Manuf., vol. 15, 2002, pp. 51-59.

[8] P.J. Timans, Advances in Rapid Thermal and Integrated Processing, edited by F. Roozeboom. Netherlands: Kluwer Academic Publishers, 1996, pp. 51-62.

[9] E. Palmer, W. Ren, C. J. Spanos and K. Poolla, "Control of photoresist properties: a kalman filter based approach", IEEE Trans. Semiconductor Manufact., vol.9, 1996, pp. 208-214.

[10] S. Leang and C. J. Spanos, "A novel In-line automated metrology for photolithography", IEEE Trans. Semiconductor Manufact., vol. 9, 1996, pp. 101-107.

[11] C. L. Henserson, S. A. Sheer, P. C. Tsiartas, B. M. Rathsack, J. P. Sagan, R. R. Dammel, A. Erdmann, and C.G.Willson, "Modeling parameter extraction for DNQ-novolac thick film resist", Proc. SPIE, vol. 3333,1998 , pp. 256-267.
[12] M. Born and E. Wolf, Principles of Optics. Oxford, U.K.: Pergamon, 1980, pp. 752-758.

[13] Y. X. Huang, "Reflection and transmission of electromagnetic waves by a dielectric medium in a arbitrary direction", J. Appl. Phys., vol. 76, no. 5, 1996, pp. 2575-2581.

[14] A. Gjurchinovski, "Reflection of light from a uniformly moving mirror," Am. J. Phys., vol. 72, no. 10, 2004, pp. 1316-1324.

[15] T. Shiozawa, K. Hazama and N. Kumagai, "Reflection and transmission of electromagnetic waves by a dielectric half-space moving perpendicular to the plate of incidence", J. Appl. Phys., vol. 38, no. 11, 1967, pp. 4459-4462 